\title{
Investigating One-Handed Multi-digit Pressure Input for Mobile Devices
}

\section{Graham Wilson}

School of Computing Science

University of Glasgow

Glasgow, G12 8QQ UK

gawilson@dcs.gla.ac.uk

\section{David Hannah}

School of Computing Science

University of Glasgow

Glasgow, G12 8QQ UK

\section{Stephen A. Brewster}

School of Computing Science

University of Glasgow

Glasgow, G12 8QQ UK

Stephen.Brewster@glasgow.ac.uk

Martin Halvey

School of Computing Science

University of Glasgow

Glasgow, G12 8QQ UK

Martin.Halvey@glasgow.ac.uk

Copyright is held by the author/owner(s)

CHI'12, May 5-10, 2012, Austin, Texas, USA.

ACM 978-1-4503-1016-1/12/05.

\begin{abstract}
This paper presents initial results from the design and evaluation of one-handed squeezing of a mobile phone: the application of force by each individual digit, and combinations of digits, of one hand as a means of interacting with a mobile device. As part of the evaluation we also consider how to alter the size of the interaction space to best suit the number of digits being used. By identifying which digits can accurately apply force both individually and in combination with others, we can then design one-handed, multi-channel input for mobile interaction. The results suggest that not all digits are equally accurate, and that some are more accurate when used in combination with others.

Further, increasing the size of the underlying interaction space to suit the number of digits used improves user performance.
\end{abstract}

\section{Author Keywords}

Pressure input; mobile interaction; one-handed interaction.

\section{ACM Classification Keywords}

H.5.2. User Interfaces: Haptic I/O.

\section{Introduction}

Mobile devices are well suited to the use of pressure as an input technique, as pressing and squeezing are a 
logical extension of common multitouch and physical gesture-based interfaces. It can add a $3^{\text {rd }}$ dimension to typically $2 \mathrm{D}$ touch interfaces as well as continuous and real-time control over content such as zooming, scrolling, panning or rotation. Research has shown pressure input on mobile devices to be highly precise when the user is sitting and walking, even using only audio feedback $[1,2]$. However most research has only used a single finger for interaction, or a single point of pressure. We have five dexterous digits available on each hand, so by understanding how precisely and how widely the digits of the hand can apply force, both individually and when used in combination, interactions can be designed to make full use of our natural ability to grip and squeeze, allowing for multiple inputs to the system. We could then potentially map more complex, two-handed interactions on to just one hand. This could be especially useful for touchscreen interaction, as common gestures require two-handed input (one to hold, one to gesture), which can be difficult to do when mobile. Beyond this, using force-sensors attached around the body of a mobile device leaves the screen free of 'fat fingers'.

\section{Pressure Input}

Some psychophysical research has suggested that precision of force output is relative to both the number of digits used and the target force to be applied [3]. Newell \& McDonald [3] found that increasing the number of digits, or "degrees of freedom", used to synchronously squeeze on a dynamometer, altered precision of force output. Specifically, precision at low levels of force degraded as more digits were added but precision at higher force levels improved. This means that our ability to control force depends on how many fingers we are using.
Pressure-based $\mathrm{HCI}$ interactions have generally consisted of only one input channel (either a single digit or single sensor) used for a single purpose, usually linear targeting. As well as the number of digits, there has also been little discussion about the influence of interaction space ('pressure space') size (normally in terms of Newtons) on pressure-based HCI. As mentioned earlier, precision in applying target forces is relative to the number of digits used. By tailoring the size of space (in Newtons) to the grip, accuracy may improve.

\section{Mobile Interaction}

While a wealth of research exists on improving touchscreen interaction, the touchscreen itself remains the most common input surface. However, during onehanded interaction, the whole surface is not easily accessible by the thumb [4], nor is the thumb necessarily the most precise pointing digit [5]. Often the contact of multiple 'fat fingers' occludes the very content with which the user is interacting. Alternative solutions include physical movement such as device tilting [6]. For the purposes of this study we looked at another alternative: using the case/body of the mobile device. By placing force sensors around the device where the fingers would naturally rest while holding it, the need for finger movement is avoided and also leaves the display entirely visible.

In this initial study, we sought to test the effects of grip (digit choice and combination) and pressure space on the precision of force application through one hand holding a mobile device as a means of interaction. 

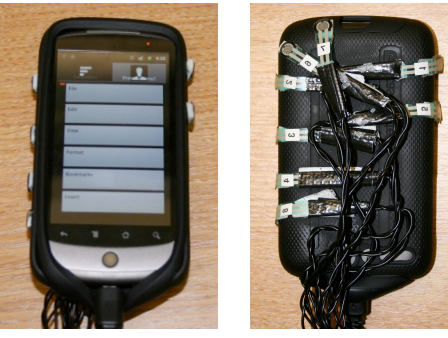

Figure 1: Sensor positions around Nexus One

\section{Experimental Design}

Force input was taken from 7 Force-Sensing Resistors (Interlink Electronics model 400FSR). These were connected to SAMH Engineering SK7-ExtGPIO01 I/O modules for analogue-to-digital conversion and sensor output linearization [2]. The two I/O modules were then connected to a MacBook Pro via USB for signal processing, which forwarded the sensor output over USB to an HTC Nexus One Android mobile phone (see Figure 1, left) to present the application GUI. The FSRs were attached to the body of the Nexus One in the configuration shown in Figure 1, for right-handed input.
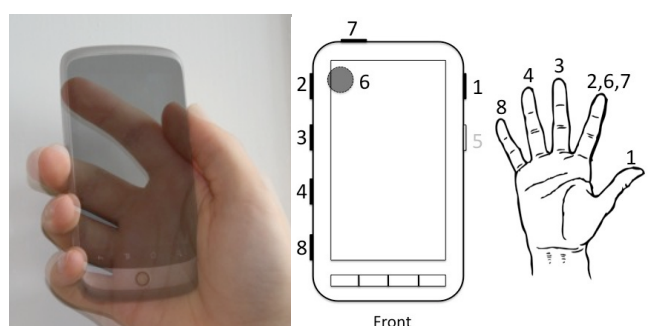

Figure 2: Common one-handed touchscreen device grip (left) and the digits used to press on each sensor (right)

The sensor positions needed to be in locations that were easily reachable. They also needed to be in positions that could provide opposing forces so that the phone could be held. One hand needed to be able to hold the device, interact with the sensors and provide opposing forces. Therefore, the most logical positions for the sensors were locations around the device near to where users naturally place fingers when holding a mobile touchscreen phone. A brief survey of these holding grips was conducted with users of touchscreen devices around the University and the most common form is shown to the left of Figure 2, in the right- handed variant. The middle $(<M>)$, ring $(<R>)$ and little $(<L>)$ finger reach round and clasp the lower lefthand side of the device, pushing it against the palm for grip and stability, leaving the thumb $(<\mathrm{T}>)$ free to interact with the screen. The index finger $(<\mathrm{I}>)$ rests along the back of the device providing further balance. From this common grip, the numbered locations shown to the right of Figure 2 were chosen for the sensors. The numbers above each digit indicate which sensors that digit pressed. They were attached to a CaseMate Tough Case (www.case-mate.com) by double-sided adhesive pads to provide extra rigidity and a flatter surface for sensor attachment.

\section{Grips}

Fourteen grip configurations were chosen in order to use each individual finger and various combinations of fingers. The grips are described in Table 1 in terms of the sensors and digits used in the grip, with the sensor numbers corresponding to those in Figure 2, right.

From the ten possible combinations of two-digit grips, G7-G9 were selected. Combinations using the thumb and one other digit were avoided for particular reasons: $<\mathrm{T}\rangle+\langle$ I $\rangle$ is a very similar grip to $<\mathrm{T}\rangle$ or $<$ I $>$ individually, as one digit opposes the other. Pressing with $\langle\mathrm{T}>$ and either $\langle\mathrm{R}>$ or $\langle\mathrm{L}>$ results in slight rotation of the device, meaning the sensors to be pressed are being pushed away from the digits pressing on them, making control more difficult. From the remaining possible choices, G7 and G9 provide grips using more adept $(<\mathrm{I}>+<\mathrm{M}>)$ and less adept $(<\mathrm{R}>+$ $<L>$ ) fingers respectively, and $G 8$ uses a combination of adept $(<\mathrm{I}>)$ and less adept $(<\mathrm{L}>)$ fingers. One three-digit grip included the fingers used to hold the phone in the common touchscreen grip (G11), with the other three-digit grip using more adept digits (G10). 


\begin{tabular}{|c|c|c|}
\hline Grip ID & Digits Used & Sensors Used \\
\hline G1 & Thumb(T) & 1 \\
\hline G2 & Middle(M) & 3 \\
\hline G3 & Ring(R) & 4 \\
\hline G4 & Index(I) & 6 \\
\hline G5 & Index(I) & 7 \\
\hline G6 & Little(L) & 8 \\
\hline G7 & I, M & 2,3 \\
\hline G8 & I, L & 2,8 \\
\hline G9 & R, L & 4,8 \\
\hline G10 & T, I, M & $1,2,3$ \\
\hline G11 & M, R, L & $3,4,8$ \\
\hline G12 & T, I, M, R & $1,2,3,4$ \\
\hline G13 & I, M, R, L & $2,3,4,8$ \\
\hline G14 & T, I, M, R, L & $1,2,3,4,8$ \\
\hline
\end{tabular}

Table 1: Grip configurations used
For each grip the whole hand was in contact with the phone, but only those sensors listed for the relevant grip took input to control the experimental software. Grips G1-G6 (see Table 1) give an indication of how precisely each individual digit can control force. Sensors 6 and 7 (in G4 and G5 respectively) introduce pressure input from the back and top of the device, positions that are not commonly used for input, and involve pressing along different planes than the sensors down the sides of the device. These will give an indication of how precise force application is when applied from different orientations.

\section{Pressure Spaces}

Two different pressure spaces were chosen, referred to here as Fixed and Incremental. In each case the input from the sensors is cumulative, so the total amount of force applied across all the sensors is taken as input. For example applying $1 \mathrm{~N}$ to each of sensors 1,2 and 3 (in $\mathrm{G} 10$ ) gives a total input to the system of $3 \mathrm{~N}$.

\section{Fixed PRessure SPACE}

Previous research on pressure input on mobile devices has used a pressure space of approximately 3.5-4N when one pressure point was used (i.e. one digit or sensor), balancing the size of interaction space with user comfort. Therefore we also used approximately $3.5 \mathrm{~N}$ as the Fixed pressure space size. Regardless of which fingers, or how many fingers, were used in a given grip the pressure space was always $3.5 \mathrm{~N}$.

INCREMENTAL PRESSURE SPACE

The use of multiple digits to apply force shifts precision from lower levels of force to higher levels [3]. From a control point-of-view, increasing the pressure space, and thereby spreading target forces over a wider range (including more target forces at higher levels) may allow for more precise control when using more digits. From an interaction point-of-view, using more fingers will increase the total interaction space available for use, so the application can make use of extra digits. Therefore, as $3.5 \mathrm{~N}$ is suitable for use when one digit is in use, the Incremental pressure space increased by $3.5 \mathrm{~N}$ with the addition of each digit, meaning $7 \mathrm{~N}$ for two digits up to $17.5 \mathrm{~N}$ for all five digits.

\section{Experimental Task}

Thirteen participants ( 6 male, 7 female) aged between 21 and 63 (mean 29.18) took part, all from within the University. Due to the positioning of the sensors, all were right-handed and each was paid $£ 10$. The experimental task was linear targeting and very similar to a common implementation $[1,7]$. The pressure space was divided into 6 equal-sized bins, visualized onscreen as a vertical menu of 6 items running from top-to-bottom measuring $465 \times 600$ pixels $(45 \times 63 \mathrm{~mm}$ see Figure 3). Each menu item also had a label (File, Edit, View, Format, Bookmarks, Insert). We chose 6 levels as it had been recommended as a suitable number for pressure interaction [1]. The position of an onscreen cursor indicated the total level of force being applied (see Figure 3 ). Each trial involved selecting a single target item by applying a target level of force, which placed the cursor within the corresponding menu item (Positional control). Items were selected by maintaining the level of force for one second ( $D$ well technique).

The study was a 2 x 14 (Pressure Space x Grip) withinsubjects design, where all participants performed all grips under both the Fixed and Incremental pressure spaces, with the order of both Grip and Pressure Space 


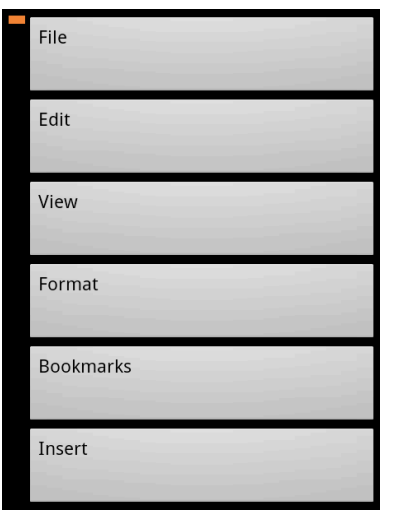

Figure 3: Interface for linea targeting task

\begin{tabular}{|c|c|c|c|}
\hline $\begin{array}{c}\text { Grip } \\
\text { ID }\end{array}$ & $\begin{array}{c}\text { ER } \\
\text { \% }\end{array}$ & $\begin{array}{c}\text { MT } \\
\text { Sec }\end{array}$ & NC \\
\hline G1 & 3.8 & $2.48(1.73)$ & $2.5(4.14)$ \\
\hline G2 & 4.8 & $2.69(2.30)$ & $3.25(5.56)$ \\
\hline G3 & 3.2 & $2.52(1.87)$ & $2.85(5.55)$ \\
\hline G4 & 9.9 & $2.24(1.42)$ & $1.30(2.37)$ \\
\hline G5 & 3.2 & $2.17(1.27)$ & $1.30(2.11)$ \\
\hline G6 & 4.8 & $2.79(2.75)$ & $3.23(5.38)$ \\
\hline G7 & 3.5 & $2.49(1.50)$ & $2.68(3.50)$ \\
\hline G8 & 6.7 & $2.54(1.66)$ & $2.65(4.26)$ \\
\hline G9 & 3.8 & $2.24(1.15)$ & $2.01(2.80)$ \\
\hline G10 & 3.5 & $2.69(2.33)$ & $3.29(6.17)$ \\
\hline G11 & 5.4 & $2.43(1.57)$ & $1.92(3.06)$ \\
\hline G12 & 7.4 & $2.52(1.66)$ & $2.41(3.68)$ \\
\hline G13 & 5.8 & $2.92(3.55)$ & $2.95(6.74)$ \\
\hline G14 & 7.1 & $2.70(2.36)$ & $2.73(5.72)$ \\
\hline & & &
\end{tabular}

Table 2: Mean results for each grip (SD in brackets) randomized. The experiment was divided up by Pressure Space: all grips were done within a pressure space before moving on to the next space. Within each grip condition every menu item was selected twice in a random order. At the start of each trial the item to be selected was highlighted in green for one second before returning to the common grey. Each Pressure Space was started with 6 practice selections. For the entire session participants were sat in a padded office chair holding the apparatus in their right hand, unsupported.

Dependent Variables were: Errors (ER: the percentage of incorrect selections), Movement Time (MT: time between first non-0 pressure reading and item selection) and Number of Crossings (NC: the number of times the cursor crossed the target's boundaries). NC is an indication of control, as a higher number of crossings indicate more erratic and uneven application of force.

\section{Results}

Due to the data violating the normality assumption, we followed the advice of Wobbrock et al. [8] and used their Aligned Rank Transform (ART) to reformat the data for use in traditional factorial analysis. For all measures we carried out a 2 x 14 Mixed Model REML (Restricted Maximum Likelihood) analysis, with 'participant' as a random factor. See Table 2 for a summary of the results.

\section{Pressure Space}

The analysis found no effect of pressure space on ER $(p>0.05)$, with means of $5.8 \%$ for the Fixed space and $4.7 \%$ for the Incremental space. There was a significant effect of pressure space on movement time (MT). The Incremental space allowed for significantly faster selections (mean=2.30s) than the Fixed space ( $m e a n=2.76 s ; p<0.001$ ). A significant effect of pressure space was also seen for measures of crossings (NC). The Incremental space resulted in significantly fewer crossings per selection (mean $=1.89$ ) than the Fixed space $($ mean $=3.12 ; \mathrm{p}<0.001)$.

Grip

The Mixed Model analysis showed a significant effect of grip on ER $(p=0.001)$. Bonferroni corrected pairwise comparisons indicated a significant difference between G4 and G3 ( $p<0.01)$, G7 $(p<0.05), G 9(p<0.01)$ and G10 ( $p<0.05)$. In all cases G4 had higher ER than the other grips. There were no other significant differences.

A significant effect of grip was also found on MT $(p<0.001)$. Bonferroni pairwise comparisons of grip showed that G4 was significantly faster than G6 $(p<0.05), G 10(p<0.05), G 13(p<0.01)$ and G14 $(p<0.05) . G 5$ was significantly faster than $\mathrm{G} 10$ $(p<0.05), G 13(p<0.01)$ and $G 14(p<0.05)$. G9 was significantly faster than $\mathrm{G} 13(\mathrm{p}<0.05)$

NC was significantly affected by grip as well $(p<0.001)$. Bonferroni comparisons showed that G4 had significantly fewer crossings than all other grips (at most $\mathrm{p}<0.05$ ) except for $\mathrm{G} 5$ and $\mathrm{G} 11$, from which $\mathrm{G} 4$ was not significantly different. Similarly G5 also had significantly fewer crossings than all other grips (at most $p<0.01$ ) other than G4, G9 and G11. G11 had significantly fewer crossings than $G 6(p<0.05)$ and $G 10$ $(p<0.01)$. G9 had significantly fewer crossings than G10 ( $p<0.01)$. The Mixed Model analysis found a significant interaction between pressure space and grip for all three measures $(p<0.001)$. 


\begin{tabular}{|c|c|c|c|}
\hline Grip ID & ER & MT & NC \\
\hline $\begin{array}{c}\text { G5 } \\
<1>\end{array}$ & $3.2 \%$ & $2.13 \mathrm{~s}$ & 1.3 \\
$\begin{array}{c}\mathbf{G 9} \\
<\mathrm{R}><\mathrm{L}>\end{array}$ & $3.8 \%$ & $2.18 \mathrm{~s}$ & 1.7 \\
$\begin{array}{c}\mathrm{G} 3 \\
<\mathrm{R}>\end{array}$ & $3.2 \%$ & $2.37 \mathrm{~s}$ & 2.4 \\
$\begin{array}{c}\mathrm{G} 7 \\
<\mid>\mathrm{M}>\end{array}$ & $3.5 \%$ & $2.38 \mathrm{~s}$ & 2.2 \\
\hline
\end{tabular}

Table 3: Best-performing grips, with Error Rate, Movement Time and Number of Crossings

\section{Discussion}

Overall, the results support the hypothesis that an increase in the number of digits used in pressure interaction would benefit from the use of a larger range of forces, as the Incremental pressure space produced better performance than the Fixed space. Therefore the interaction space should be tailored to the number of digits used. Concerning grips, there were several interesting results. Firstly, not all digits were equally accurate when used individually, suggesting that 5channel input, one per finger, is not necessarily feasible. However those fingers that did not perform well performed better when used in concert with other digits, so variations of pressure 'chording' could be used as well. Although it might be expected that the index finger $(<\mathrm{I}>)$ would be highly precise, this was not always the case. When pressing from the top, $<$ I $>$ had the highest accuracy of all grips, whereas, when pressing from the back, it had the lowest accuracy. The combination of $\langle$ I $>$ and $\langle$ M $>$ performed well but $<$ I $>$ combined with $<L>$ performed poorly. Somewhat surprisingly $<R>$ had low errors and good precision in isolation as well as in combination with $\langle\mathrm{L}\rangle$, and $\langle\mathrm{M}\rangle$ $\&<L>$. The results indicated that the grips using $\langle T\rangle$ as well as 3 or 4 other digits performed poorly.

Table 3 shows the overall best-performing digits/grips. Using just these, four different inputs could be used to control a mobile device. A further step we will take is to test control using the other digit combinations omitted here. Also, we have only included the portrait orientation here; it would be necessary to understand how different device orientations will influence grip and control using those grips. Once we have a fuller picture of multi-digit control we plan to compare common twohanded input, such as those requiring multitouch gestures (e.g. zooming and rotating), to severa alternative one-handed techniques including multi-digit pressure. Aside from multi-channel input, the benefits that pressure input could bring include continuous, analog input as well as the avoidance of screenocclusion.

\section{References}

[1] Ramos, G., Boulos, M. and Balakrishnan, R. Pressure widgets. In Proceedings of CHI 2004, p. 487-494.

[2] Wilson, G., Brewster, S. A., Halvey, M., Crossan, A.

and Stewart, C. The Effects of Walking, Feedback and Control Method on Pressure-Based Interaction. In Proceedings of MobileHCI 2011, p. 147-156.

[3] Newell, K. and McDonald, P. Information, coordination modes and control in a prehensile force task. Human Movement Science, 13, 3-4 (1994), 375-391.

[4] Perry, K. and Hourcade, J. P. Evaluating One Handed Thumb Tapping on Mobile Touchscreen Devices. In Proceedings of Graphics Interface 2008, p. 57-64.

[5] Wobbrock, J., Myers, B. and Aung, H. The performance of hand postures in front-and back-of-device interaction for mobile computing. International Journal of HumanComputer Studies, 66 (2008), 857-875.

[6] Eslambolchilar, P. and Murray-Smith, R. Control centric approach in designing scrolling and zooming user

interfaces. International Journal of Human-Computer Studies, 66 (2008), 838-856.

[7] Cechanowicz, J., Irani, P. and Subramanian, S. Augmenting the mouse with pressure sensitive input. In Proceedings of CHI 2007, p. 1385-1394.

[8] Wobbrock, J., Findlater, L., Gergle, D. and Higgins, J. The Aligned Rank Transform for nonparametric Factorial Analyses Using Only AVOVA Procedures. In Proceedings of CHI 2011, p. 143-146. 